\title{
Analysis of photon scattering trends for material classification using artificial neural network models
}

\begin{abstract}
In this project, we concentrate on using the Artificial Neural Network (ANN) approach to analyze the photon scattering trend given by specific materials. The aim of this project is to fully utilize the scatter components of an interrogating gamma-ray radiation beam in order to determine the types of material embedded in sand and later to determine the depth of the material. This is useful in a situation in which the operator has no knowledge of potentially hidden materials. In this paper, the materials that we used were stainless steel, wood and stone. These moderately high density materials are chosen because they have strong scattering components, and provide a good starting point to design our ANN model. Data were acquired using the Monte Carlo N-Particle Code, MCNP5. The source was a collimated pencil-beam projection of $1 \mathrm{MeV}$ energy gamma rays and the beam was projected towards a slab of unknown material that was buried in sand. The scattered photons were collected using a planar surface detector located directly above the sample. In order to execute the ANN model, several feature points were extracted from the frequency domain of the collected signals. For material classification work, the best result was obtained for stone with $86.6 \%$ accurate classification while the most accurate buried distance is given by stone and wood, with a mean absolute error of 0.05 .
\end{abstract}

Keyword: Artificial neural network (ANN); Depth determination; Material classification; MCNP; Stainless steel; Wood 